\title{
Revisão de dois instrumentos clínicos de avaliação para predizer risco de quedas em idosos
}

\author{
Review of two clinical assessment tools to predict risk of falls in elderly
}

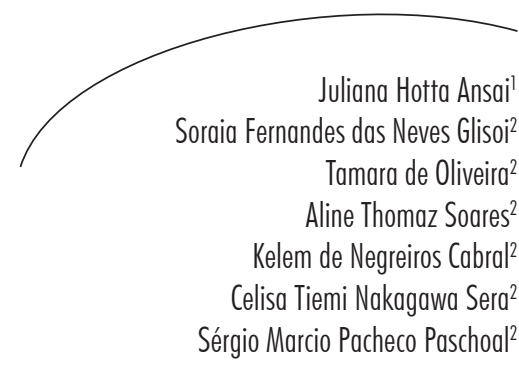

Resumo

Introdução: Quedas são um problema clínico comum nos idosos, que pode reduzir sua mobilidade e independência. O uso de instrumentos simples para detecção do risco de quedas é fundamental para prevenção e tratamento de tais eventos. Não há, porém, consenso quanto aos testes mais adequados para cada situação. Objetivo: Revisar estudos sobre eficácia, sensibilidade e especificidade dos testes Timed Up and Go Test e Berg Balance Scale, a fim de verificar qual é o mais apropriado para predizer quedas em idosos. Métodos: Realizou-se revisão bibliográfica nas bases de dados MEDLINE, PubMed, ISI, LILACS e Portal de Periódicos CAPES, entre os anos de 2001 e 2011. Resultados: Foram selecionados 37 artigos, sendo 17 sobre a Berg Balance Scale e 20 sobre o Timed Up and Go Test. A revisão mostrou que os dois testes podem ser bons preditores de quedas, mas os artigos diferiram quanto à definição de queda e caidor, tipo de estudo, quantidade e característica da amostra e avaliação de quedas, levando a diferentes resultados quanto a nota de corte, sensibilidade, especificidade e predição de quedas. Há controvérsias quanto à capacidade de predição em perfis específicos, como os idosos ativos. Conclusão: Os testes avaliados são eficazes para predição de quedas, desde que adaptados para cada perfil. Novos estudos devem ser realizados com metodologia homogênea, a fim de favorecer a comparação de resultados sobre a eficácia desses testes.

\section{Abstract}

Introduction: Fall is a common problem in the elderly and it can reduce their mobility and independence. The use of simple tools to detect risk of falls is essential to prevent and treat such events. However, there is no consensus about the most appropriate tools for each situation. Objective: To review studies about efficacy, sensitivity and specificity of Timed Up and Go Test (TUGT) and Berg Balance Scale (BBS) in order to ascertain which is the most appropriate to predict falls in the elderly. Methods: We performed a literature review from MEDLINE, PubMed, ISI, LILACS and Portal de Periódicos

\footnotetext{
Programa de Pós-graduação em Fisioterapia. Universidade Federal de São Carlos. São Carlos, SP, Brasil.

Hospital das Clínicas, Faculdade de Medicina. Universidade de São Paulo. São Paulo, SP, Brasil.

Correspondência / Correspondence

Juliana Hotta Ansai

E-mail: juansai@msn.com
}

Palavras-chave: Idoso.

Acidentes por Quedas.

Quedas.Timed Up and Go. Berg

Balance Scale. 
CAPES databases, between 2001 and 2011. Results: We selected 17 different articles about BBS and 20 articles about TUGT. The review showed the two tools can be good predictors of falls. However, the articles differed in definition of fall and faller, type of study, quantity and characteristics of sample and assessment of falls, leading to different results as cutoff scores, sensitivity, specificity and prediction of fall. There is controversy about the ability of tools to predict falls in specific samples, such as active elderly. Conclusion: TUGT and BBS are effective to predict falls, provided they are adapted to each sample. Further studies should be performed using articles with homogeneous methods in order to support comparison of results about the effectiveness of tools.
Key words: Elderly. Falls. Timed Up and Go. Berg Balance Scale.

\section{INTRODUÇÃO}

Quedas são um problema clínico comum na população idosa. No Brasil, cerca de $30 \%$ dos idosos caem pelo menos uma vez ao ano. ${ }^{1}$ A queda é mais frequente em mulheres ${ }^{1}$ e sua ocorrência aumenta com a idade, sendo que $32 \%$ dos idosos entre 65 e 74 anos, 35\% de 75 a 84 anos e $51 \%$ acima de 85 anos de idade caem por ano. $^{2}$ Entre 5 e $10 \%$ dos idosos residentes na comunidade com histórico de quedas apresentam graves consequências, como fratura, traumatismo craniano e lacerações sérias. Com isso, há a possibilidade de reduzir sua mobilidade e independência, aumentando as chances de morte prematura. Cerca de metade dos idosos hospitalizados por fratura de quadril não recuperam a mobilidade prévia ao evento. ${ }^{3}$

Queda em idosos é alvo de questões de saúde pública e a capacidade de profissionais da área da saúde em detectar risco de quedas e fatores de risco modificáveis, por meio de instrumentos de triagem simples, é um elemento fundamental na sua prevenção. ${ }^{4}$ Ainda não há, no entanto, consenso quanto aos instrumentos mais adequados na detecção de risco de quedas em cada situação, o que gera dificuldades tanto na avaliação quanto na escolha da intervenção mais adequada para prevenir tais eventos..$^{5-7}$

Em 2006, especialistas alemães em prevenção de quedas avaliaram os instrumentos existentes para detectar risco de quedas de forma crítica. Dois aspectos foram em especial interessantes: a carência de informações sobre sensibilidade, especificidade, confiabilidade e validade preditiva em diferentes populações e o tempo adicional necessário para esse tipo de triagem em assistências de rotina. ${ }^{8}$

No presente estudo, foram revisados dois testes funcionais, Berg Balance Scale (EEB) e Timed Up and Go Test (TUGT), os quais foram escolhidos por serem de fácil aplicação, baixo custo e utilizados em pesquisas e na prática clínica.

A EEB avalia o desempenho do equilíbrio funcional em 14 itens comuns da vida diária numa ordem padrão. Cada tarefa é pontuada entre 0 e 4 pontos, de acordo com a qualidade do desempenho ou o tempo necessário para completar a tarefa, sendo que 0 indica incapacidade de realizar, e 4, realização com independência. A pontuação máxima é de 56 pontos, relacionada a um bom equilíbrio. ${ }^{9}$

O TUGT avalia mobilidade e equilíbrio, ${ }^{6}$ sendo que a identificação de pacientes com risco de quedas baseada nesses itens é muito relevante para que uma correta intervenção seja introduzida, mas não se sabe ao certo em qual tipo de população o teste teria melhor proveito.

Dada a utilização de diversos instrumentos para avaliação de quedas em idosos, o presente estudo teve como objetivo revisar os estudos sobre eficácia, sensibilidade e especificidade dos instrumentos Berg Balance Scale (EEB) e Timed Up and Go Test (TUGT) para predizer quedas em idosos, a fim de verificar qual instrumento é mais apropriado para tal detecção, ajudando na prevenção e no tratamento de tais eventos. 


\section{METODOLOGIA}

Foi realizada revisão bibliográfica quantitativa da literatura nacional e internacional. A partir das palavras-chave "Berg balance scale", "elderly", "fall" e "timed up and go test" e os termos correspondentes em português, foram identificados 878 artigos publicados entre os anos de 2001 a 2011; destes, foram excluídos 841 , sendo selecionados para a pesquisa 37 artigos publicados nos idiomas inglês e português. Os artigos consultados foram obtidos por meio da pesquisa eletrônica nas bases de dados MEDLINE, PubMed, ISI, LILACS e Portal de Periódicos CAPES, e pela consulta a artigos indicados nas referências dos artigos científicos identificados na pesquisa.

A partir da busca de artigos pelas palavraschave, foram selecionados os que estudaram uma população acima de 60 anos de idade, residentes na comunidade e que tinham como um dos objetivos avaliar ou comparar os testes em relação ao risco de quedas. Artigos que utilizaram populações com doenças específicas, exceto síndrome da fragilidade, por se tratar de um acometimento comum na população idosa da comunidade, foram excluídos do trabalho. Ainda, artigos com restrição de acesso, sem possibilidade de serem encontrados no Portal de Periódicos CAPES, foram excluídos.

Os 37 artigos selecionados foram avaliados por três pesquisadores. Caso algum dos pesquisadores tivesse dúvida quanto à adequação do artigo para a revisão, era feito um consenso entre os avaliadores. A partir dos 37 artigos, os instrumentos foram analisados separadamente e então comparados, a fim de verificar qual instrumento era mais apropriado para predizer quedas em idosos da comunidade.

\section{RESULTADOS}

Foram revisados 37 artigos: Timed Up and Go Test $(\mathrm{n}=20)$ e Berg Balance Scale $(\mathrm{n}=17)$. Um artigo publicado em $1996^{10} \mathrm{e}$ outro publicado em
$1992^{11}$ foram selecionados devido a sua grande relevância bibliográfica. Três artigos estavam no idioma português ${ }^{9,12,13}$ e um no idioma espanhol. ${ }^{10}$ Dos 37 artigos revisados, nove compararam os dois testes. ${ }^{6,9,14-20}$

\section{Definição de queda}

Dos 17 artigos revisados sobre a EEB, apenas quatro definiram quedas, porém nenhuma definição foi semelhante. . $^{1021-23}$ Com relação ao TUGT, quatro artigos do total de 20 não definiram queda, ${ }^{6,9,24,25}$ mas houve sete definições distintas para o evento dentre os 16 artigos. A definição mais utilizada nos artigos da revisão completa foi "uma mudança não intencional da posição do corpo, resultando em contato com o solo ou outro nível inferior, no entanto, não em consequência de um evento intrínseco importante (como acidente vascular cerebral, síncope) ou um grande perigo (como acidente de carro)". $15,18,23,26-28$

\section{Objetivo e descrição dos instrumentos}

A EEB foi desenvolvida e validada por Berg et al. em 1989, com tradução e adaptação transcultural para o Brasil em 2004. ${ }^{11,12,29}$ A validade do conteúdo da EEB foi criada numa terceira fase, com 32 profissionais peritos em trabalho no contexto geriátrico. ${ }^{30}$

A escala foi desenvolvida para monitorar quantitativamente o desempenho dos idosos frágeis e pacientes com distúrbios de equilíbrio quanto à realização de tarefas motoras da vida diária e desafiadoras. $\mathrm{Na}$ prática clínica, pontuações abaixo de 45 pontos indicam risco de quedas. ${ }^{10,21,22,29}$ Para aplicação do teste, são necessários instrumentos como: step ou degrau de escada, cadeira com e sem braços, fita métrica, cronômetro, caneta e uma mesa. ${ }^{10,13,29}$ No quadro, os itens da EEB são apresentados. 
Quadro 1. Escala de Equilíbrio de Berg. São Paulo-SP, 2012

\begin{tabular}{|c|l|}
\hline Item & Descrição \\
\hline 1 & Sentado para em pé \\
\hline 2 & Em pé sem apoio \\
\hline 3 & Sentado sem apoio \\
\hline 4 & Em pé para sentado \\
\hline 5 & Transferências \\
\hline 6 & Em pé de olhos fechados \\
\hline 7 & Em pé com os pés unidos \\
\hline 8 & Avançando com braço estendido \\
\hline 9 & Recuperando objeto no chão \\
\hline 10 & Virando-se para olhar para trás \\
\hline 11 & Giro de $360^{\circ}$ \\
\hline 12 & Tocar os pés alternadamente em um step \\
\hline 13 & Um pé na frente do outro (tandem) \\
\hline 14 & De pé sobre uma perna (unipodal) \\
\hline
\end{tabular}

Amaioria dos artigos descreveu oTUGT como levantar-se de uma cadeira, andar por três metros, virar-se, voltar em direção à cadeira e sentar-se, sendo o tempo mensurado. , $9,14-17,25,26,28,31,32$ Além destas instruções, outros pediam ao indivíduo para ficar com o tronco encostado na cadeira inicialmente ${ }^{33,34}$ e não utilizar os braços para se levantar. ${ }^{18}$ Apenas dois artigos relataram ter colocado uma marca no chão no momento de virar-se. ${ }^{19,34}$ Huang et al. ${ }^{35}$ utilizaram um percurso de dez metros. Três artigos não descreveram o teste. ${ }^{7,24,27}$

Alguns artigos realizaram modificações do TUGT convencional. Greene et al. ${ }^{19}$ realizaram o teste associado ao uso de sensores cinemáticos para visualização de parâmetros derivados.
Hofheinz \& Schusterschitz ${ }^{6}$ realizaram o TUGT associado a uma tarefa manual (TUGTman) e cognitiva (TUGTcog), a fim de verificar o grau de automatismo do movimento. No TUGTman, deve-se realizar o teste segurando um copo $(8 \mathrm{~cm}$ de diâmetro e altura $9,5 \mathrm{~cm})$ cheio de água $(1 \mathrm{~cm}$ abaixo da borda do copo) localizado inicialmente em uma mesa de altura $70 \mathrm{~cm}$. No TUGTcog, o indivíduo realiza o percurso associado à tarefa de contar de trás para frente de três em três. A contagem começou em vários pontos, a fim de excluir efeitos de treinamento.

Garriga et al. ${ }^{36}$ utilizaram o TUGT associado a uma tarefa física e cognitiva. O indivíduo é orientado a deixar o tronco apoiado na cadeira, 
levantar-se sem usar os braços, chutar uma bola localizada a frente (diâmetro $19 \mathrm{~cm}$ e 0,2 kg) o mais forte possível, andar na sua velocidade usual por seis metros contando de 15 a 0 inversamente, virar-se pelo cone sem tocá-lo e andar pisando em círculos até sentar-se na cadeira. Além do tempo mensurado em cada estágio, uma avaliação qualitativa de 0 (necessidade de ajuda para realizar a tarefa) a 3 (performance sem ajuda e sem erros) foi feita.

Com relação à velocidade, oito artigos não especificaram a orientação. ${ }^{7,9,14,15,19,27,35} \mathrm{~A}$ maioria dos estudos orientou uma velocidade usual, ${ }^{17,18,25,26,28,31,32,34,36}$ apesar de três estudos terem instruído uma marcha mais rápida possível e com segurança. ${ }^{16,19,33}$ Sobre a dimensão da cadeira, seis trabalhos utilizaram uma cadeira de altura entre 44 e 47 cm. ${ }^{6,16,18,19,33,34}$ Somente quatro artigos relataram utilizar cadeiras com descanso de braço, ${ }^{16,19,33,34}$ sendo que apenas dois especificaram sua altura em relação ao chão $\left(65,41 \mathrm{~cm}^{16}\right.$ e $\left.65 \mathrm{~cm}^{19}\right)$.

Apenas um especificou quanto ao tipo de sapato (usual) ${ }^{7}$ e quatro relataram realizar o teste com o dispositivo de marcha usual (bengala, andador), caso o paciente fizesse uso deste dispositivo. ${ }^{7,18,26,28}$ Para o paciente se familiarizar com o teste, cinco trabalhos relataram realizar um pré-teste. ${ }^{6,7,14,17,32}$ Quatro utilizaram, como valor válido, a média de dois resultados. 17,18,30,31
EEB - confiabilidade, nota de corte,

sensibilidade (S) e especificidade (E)

Estudos encontraram boa confiabilidade interexaminadores (ICC-0,98) eintraexaminadores (ICC-0,99), com consistência interna de 0,96. Berg et al. ${ }^{12}$ realizaram a validade concorrente com as escalas POMA ( $r=0,91)$, Barthel $(r=0,67)$, TUGT $(\mathrm{r}=0,76)$, Balance Máster $(\mathrm{r}=0,81)$. Em 1996, foi encontrada uma confiabilidade interexaminador de $88 \%$ quando os avaliadores eram informados com instruções escritas e autorizados a fazer perguntas antes de administrar a EEB.${ }^{10}$ Resultados semelhantes foram encontrados por Wang Cy et al. ${ }^{20}$ em 268 idosos apresentando boa aceitabilidade dos escores da escala, boa consistência interna e confiabilidade interexaminador. A tabela 1 mostra as características e resultados dos estudos da EEB.

TUGT - confiabilidade, nota de corte, sensibilidade (S) e especificidade (E)

Alguns estudos mostraram boa confiabilidade intraexaminadores $(\mathrm{ICC}=0,75)^{7}$, confiabilidade intra e interexaminadores excelente $(\mathrm{ICC}=0,93$ a 0.99, em 1.200 idosos), a qual não diferiu conforme o status cognitivo. ${ }^{25}$ A tabela 2 mostra as características e resultados dos estudos do TUGT. 


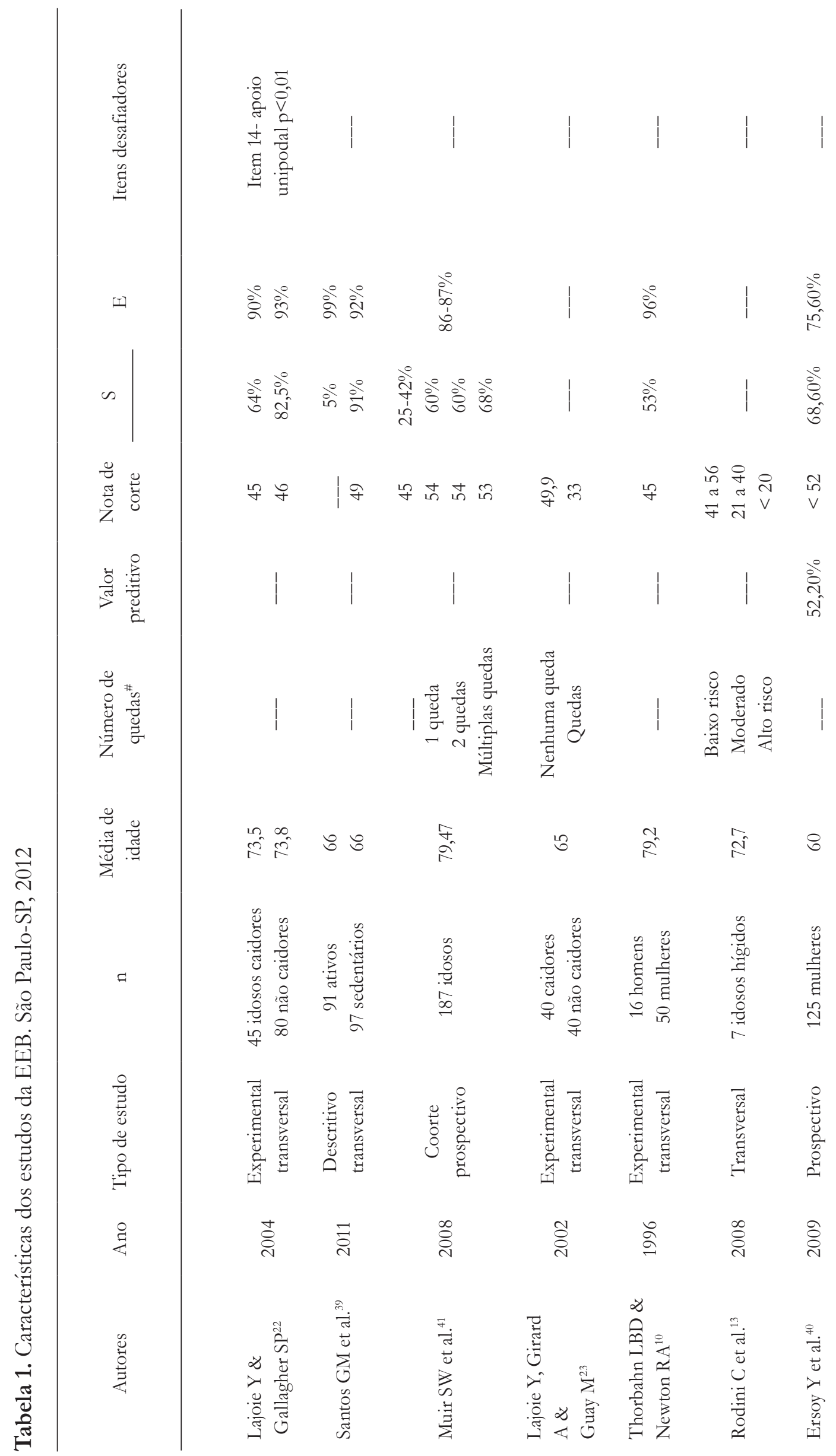



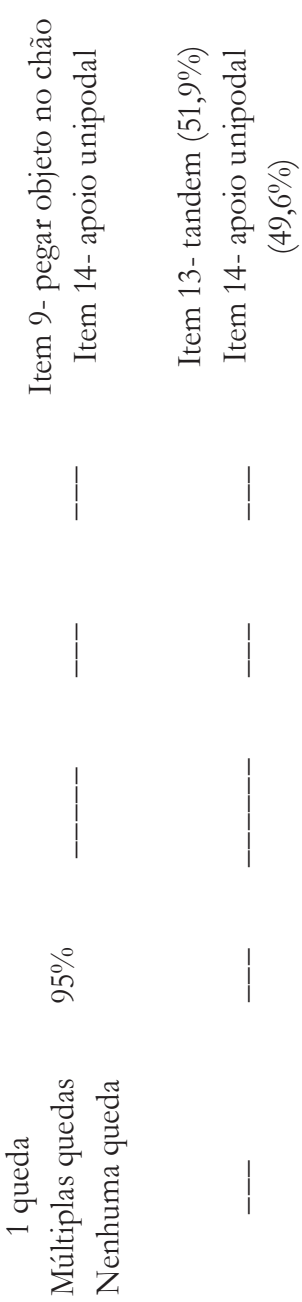

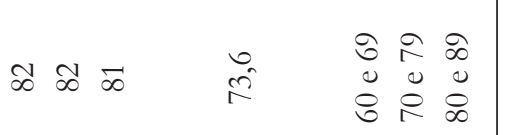

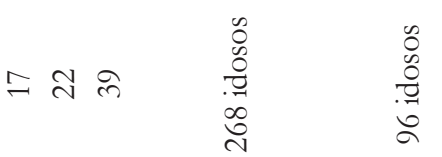

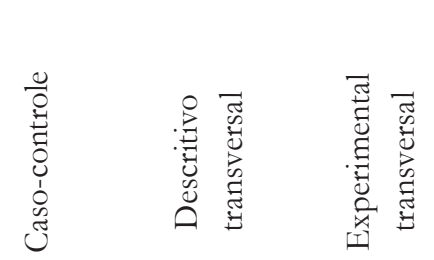

๙ิڤ

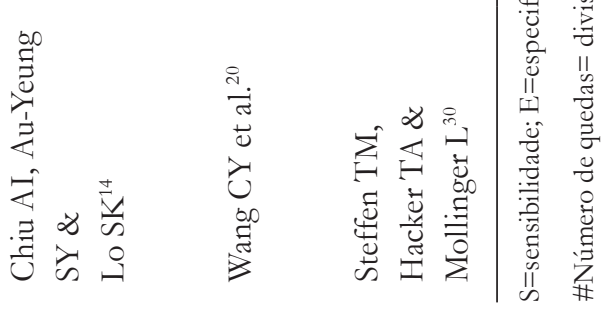

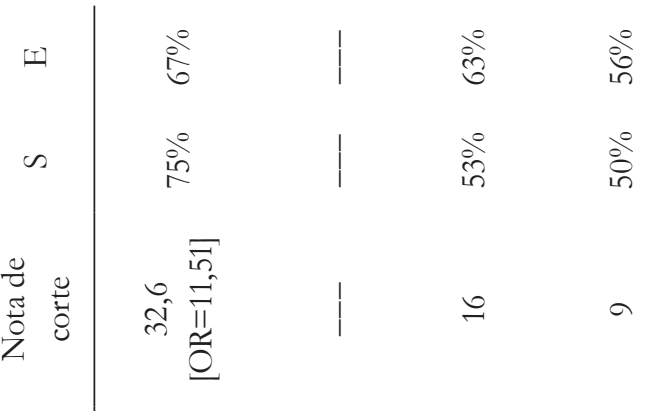

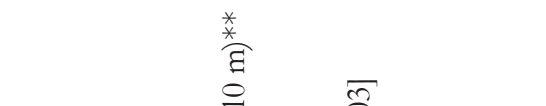

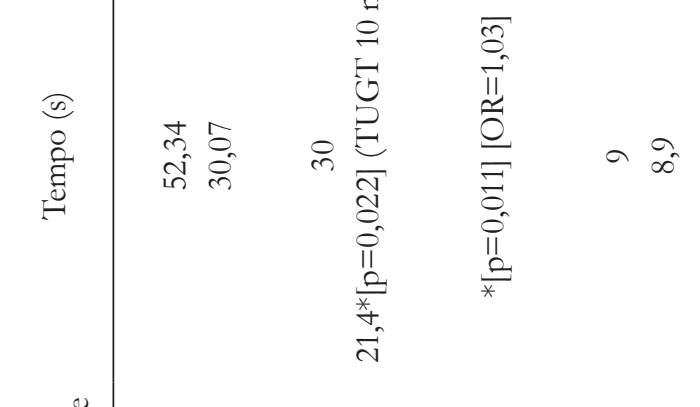

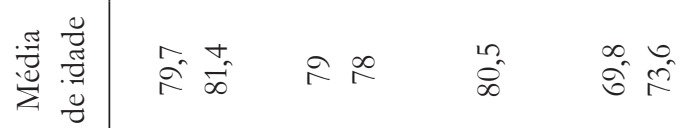

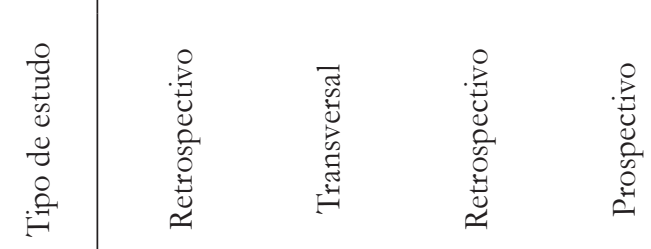

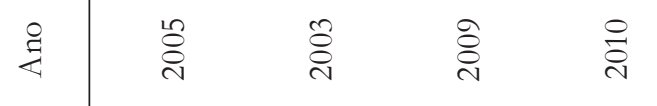

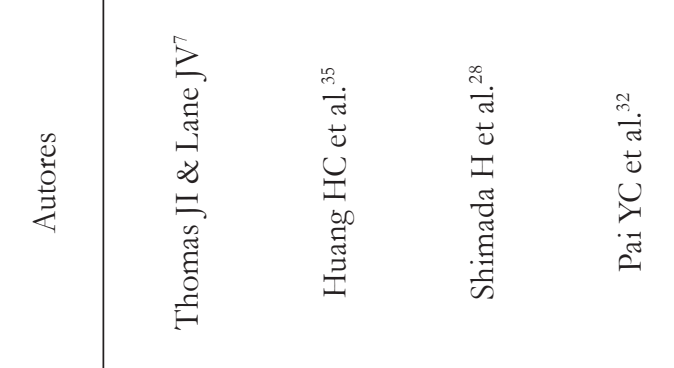




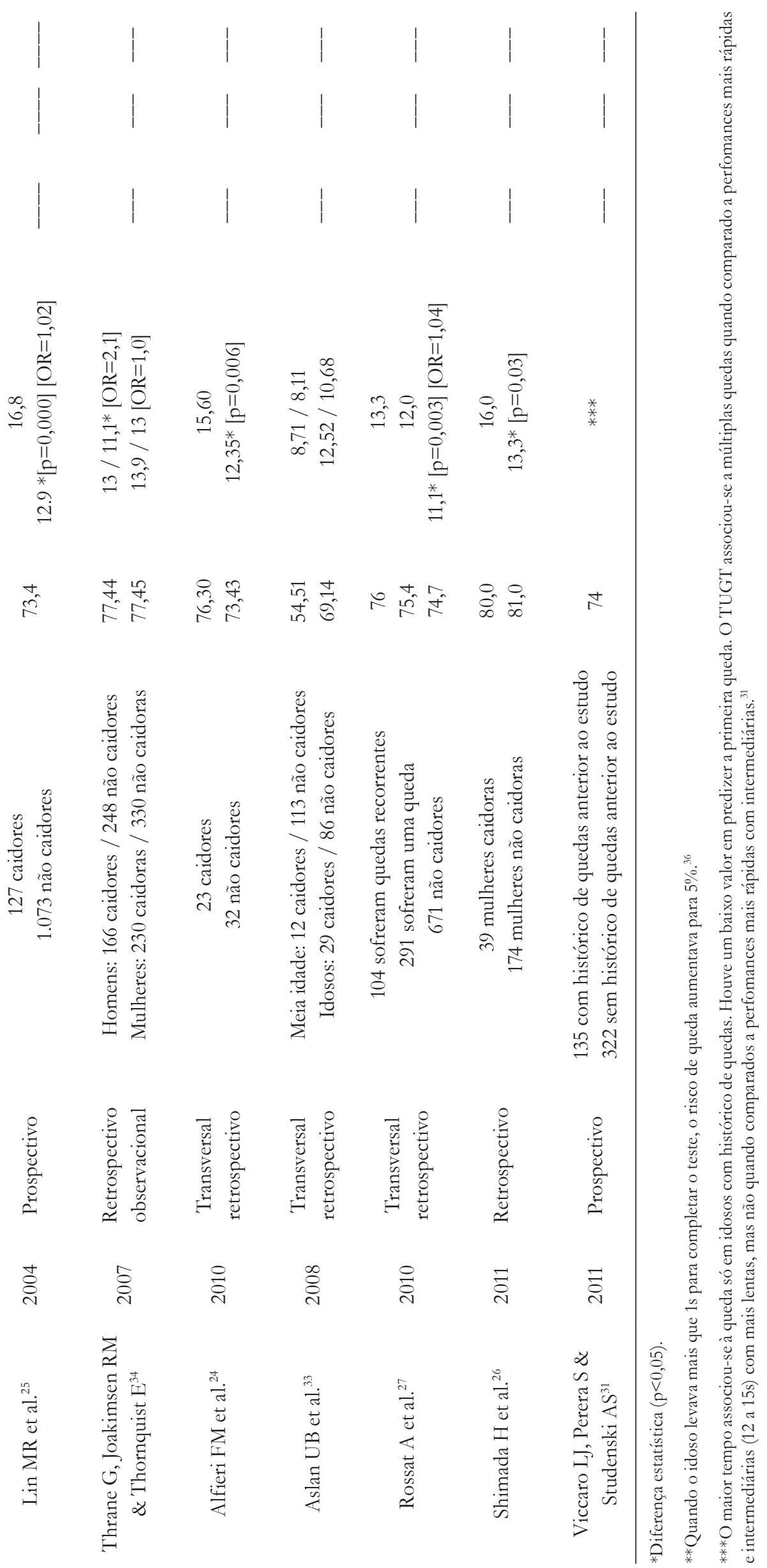


Modificações no TUGT

Na Alemanha, 120 idosos de um centro de fisioterapia realizaram os testes EBS, TUGT, TUGTman e TUGTcog, a fim de validar os testes com dupla tarefa (DT). Como não havia histórico de quedas atualizado, os testes foram comparados à pontuação da EBS. A performance entre o TUGT e o TUGT-DT não diferiu significativamente entre os sexos, porém houve diferença entre as faixas etárias. A correlação entre o TUGT e o TUGTman com a EBS foi alta $(0,74$ e 0,72 , respectivamente). Apesar de o TUGTcog não apresentar coeficiente tão alto com a EBS $(0,66)$, houve bom critério de validação em predizer risco de quedas nos testes de DT. A confiabilidade teste-reteste nos dois testes de DT foram muito boas (acima de 0,97). A confiabilidade intra-avaliadores foi muito alta (ICC: TUGTman $=0,99$; TUGTcog $=0,94){ }^{6}$

Estudo retrospectivo foi realizado em 349 idosos para verificar a associação entre o risco de quedas com TUGT, EBS e TUGT com sensores cinemáticos. Cada sensor continha um acelerômetro triaxial e uma borda giroscópia triaxial. A média do TUGT em pacientes com histórico de quedas autorrelatado nos últimos cinco anos foi de $11,5 \pm 5,2 \mathrm{~s}$, e sem histórico de quedas foi de $8,5 \pm 2,6 \mathrm{~s}$ ( $\mathrm{S}=58 \%$; $\mathrm{E}=64,8 \%$ ). $\mathrm{Na}$ EBS, a média foi de $49,8 \pm 7,0$ e $54,0 \pm 3,1$, respectivamente $(S=57,8 \% ; E=64,2 \%)$. A EBS e o TUGT foram negativamente correlacionados. Dos 44 parâmetros derivados dos sensores, 29 obtiveram discriminação significativa entre caidores e não caidores ( $\mathrm{S}=77,3 \% ; \mathrm{E}=75,9 \%$ ), mostrando que os sensores podem quantificar objetivamente o TUGT e melhorar a análise de cada segmento do teste. ${ }^{19}$

Garriga et al. ${ }^{36}$ avaliaram a sensibilidade de um TUGT modificado em predizer quedas em idosos. Para verificar a confiabilidade do teste, foram recrutados dez idosos (dois homens e oito mulheres, média 83,4 anos) que moravam num residencial geriátrico, capazes de andar sem dispositivo de marcha e seguir ordens simples. O histórico de quedas em seis meses foi coletado por meio de autorrelato e entrevista com o médico. Os idosos realizaram o teste duas vezes por três examinadores diferentes para verificar a confiabilidade entre avaliadores.

Ainda nesse mesmo estudo, com o objetivo de avaliar a concordância entre os três investigadores, os coeficientes de confiabilidade, coeficientes de correlação intergrupos e limites de concordância, 60 idosos foram recrutados (51 mulheres e nove homens, média 74,2 anos) para fazer parte da comparação entre grupos, sendo: 15 sedentários que tinham ao mínimo uma queda nos últimos seis meses (G1), 15 sedentários sem histórico de quedas (G2), 15 ativos (moderado/alto nível de atividade física baseado no questionário de autorrelato IPAQ) que sofreram quedas (G3) e 15 ativos sem histórico de quedas (G4). O coeficiente de confiabilidade no tempo total do teste foi acima de 98\%, com ICC $=0,999$. A diferença no tempo total entre os três avaliadores foi de 0,19 a 0,55s. Através do Kappa $\mathrm{K}$ de Cohen, o teste demonstrou alta confiabilidade interexaminadores. $\mathrm{Na}$ comparação intergrupos, houve diferença significativa no tempo total entre o G4 e os outros grupos, e entre G2 e G1, demonstrando boa sensibilidade para detectar risco de quedas em idosos. ${ }^{36}$

\section{DISCUSSÃO}

Tanto o TUGT e a EEB vêm sendo amplamente utilizados nas pesquisas relacionadas ao equilíbrio em idosos. No entanto, ainda há controvérsias sobre seu poder de predição de quedas, vantagens e desvantagens. Dentre as vantagens, destacam-se: são simples, de fácil administração e aplicação, exigem poucos materiais de baixo custo, além de seguros para aplicação em idosos. No caso do TUGT, mesmo com dupla tarefa, consome menor tempo quando comparado a EEB que leva de 15 a 20 minutos para ser aplicada. ${ }^{10,13,29,37}$

O TUGT é o único teste recomendado pela American and British Geriatrics Societies para avaliação de risco de quedas, ${ }^{16}$ incluindo itens funcionais com alta relação ao número de quedas como um componente de marcha importante, 
devido a ocorrência de quedas durante a deambulação ${ }^{7}$ e dupla tarefa, associada a situações diárias envolvendo demandas simultâneas. ${ }^{6}$ Não apresenta efeito teto 9 e é um teste objetivo devido à cronometragem do tempo para execução da tarefa, o que o torna mais sensível como instrumento.?

A EEB, por sua vez, limita-se pela falta de um teste que avalie a execução de múltiplas tarefas ${ }^{38}$ fazendo uso da observação do avaliador para a análise do desempenho da atividade. ${ }^{9}$ Estudos têm relatado que esta escala não é totalmente representativa em relação à sua previsibilidade de conclusão de tarefas mais complexas ou atividades que exijam controle postural e ajustes antecipatórios. ${ }^{22}$ As propriedades psicométricas de confiabilidade e validade desta escala têm sido bem demonstradas, sendo considerado um teste padrão ouro para o risco de quedas. É amplamente utilizado para identificar indivíduos que se beneficiariam de um encaminhamento para fisioterapia e para prever quedas em idosos da comunidade e institucionalizados. ${ }^{9,21,22,29,39}$

Há grande divergência entre os estudos quanto às notas de corte das escalas. Isso ocorre devido à heterogeneidade na seleção dos idosos pesquisados, como a ampla faixa etária e os níveis diferenciados de funcionalidade, ou seja, desde idosos sedentários, ativos, com distúrbios de marcha e/ou equilíbrio, além do fato de alguns necessitarem do uso de dispositivos auxiliares de marcha. É importante igualar esses fatores, já que estudos apontam o gênero feminino e o aumento da idade como fatores de risco com grande relevância para a ocorrência de quedas em idosos. Na prática clínica, a utilização dessas notas de corte, sem considerar essa diversidade, pode resultar em prognósticos individuais errôneos e, consequentemente, tratamentos inadequados. ${ }^{40}$

Shimada et al. recrutaram idosos frágeis (presença de fraqueza, baixa atividade física e/ou velocidade de marcha baixa) de um centro-dia, onde o TUGT mostrou predizer quedas significativamente, mas os autores não consideraram um teste válido clinicamente devido ao OR próximo a zero. ${ }^{28} \mathrm{Lin}$ et al. mostraram que o TUGT pôde predizer a ocorrência de quedas, mesmo incluindo ou não o status cognitivo. ${ }^{25}$ Alfieri et al. encontraram diferença significativa no tempo de execução do teste entre caidores e não caidores, porém um maior número de não caidores realizava atividade física moderada, comparado aos caidores. ${ }^{24}$

Aslan et al. demonstraram que os indivíduos de meia idade, assim como os homens, tiveram melhores pontuações no TUGT, em comparação aos idosos e mulheres. Não houve diferença significativa entre caidores e não caidores, apesar de os idosos caidores tenderem a piores pontuações. ${ }^{33}$ Rossat et al. mostraram que o número de quedas aumentou significativamente com o tempo do TUGT, mas após ajuste por idade e gênero, o tempo do TUGT foi significativamente associado somente com quedas recorrentes, e não com um único evento. Após ajustar por potenciais confundidores (modelo backward stepwise), não houve associação com quedas. ${ }^{27}$

Devido ao efeito teto da EEB em idosos ativos, são necessários altos valores de corte para otimizar a sensibilidade em cada categoria. Isso indica que o comprometimento do equilíbrio por si só não define risco de queda aumentado e que as quedas são frequentes entre as pessoas com pontuações acima de 45. O uso desses escores mais altos de corte mudaria a definição operacional de comprometimento equilíbrio, de modo que algumas pessoas definidas como tendo disfunção apresentariam uma organização independente $\mathrm{e}$ funcional com uma gama de habilidades de equilíbrio. Mesmo com os maiores escores de corte, a sensibilidade seria subótima para um contexto clínico. ${ }^{22,39,41}$ Wang et al. observaram que em quase todas as questões da EEB se obteve ponto máximo. Observouse que a soma dos itens 8, 13 e 14 apresentou influência significativa na mobilidade e nas atividades instrumentais de vida diária entre 
idosos saudáveis e comprometidos. ${ }^{20} \mathrm{O}$ modo de fornecimento do histórico de quedas (autorrelato, contato telefônico, diário de quedas) e a quantidade de meses coletados divergiram entre os estudos, o que pode ter gerado um número de quedas sub/superestimado. ${ }^{15,34}$

Diversos estudos foram realizados com o intuito de comparar EEB e TUGT na predição de quedas em idosos. Observa-se grande divergência quanto ao melhor teste utilizado e quanto à correlação entre os testes, devido à variação das amostras estudadas.

Um estudo comparou' a EEB e o TUGT em idosos sem histórico de quedas (grupo 1), com uma queda (grupo 2) e quedas recorrentes (grupo 3), com 32 idosos em cada grupo. A média no TUGT foi $11,43 \pm 2,95 \mathrm{~s}$ para o grupo 1 , $14,57 \pm 4,23$ s para o grupo 2 e $14,48 \pm 4,46$ s para o grupo 3. A diferença de médias foi significativa entre os grupos 1 e $2(p=0,005)$ e entre os grupos 1 e 3 ( $p=0,007)$. Na EEB, idosos com histórico de quedas recorrentes apresentaram declínio do equilíbrio comparado aos idosos sem quedas (grupo 1 foi de 52,00 $\pm 3,60$ pontos, a do grupo

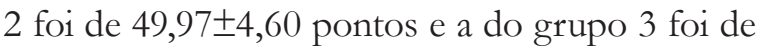
$48,91 \pm 4,55$ pontos). Para a nota de corte 45 , não foram encontradas diferenças entre os grupos, a diferença foi significativa entre os grupos $1 \mathrm{e}$ $3(\mathrm{p}=0,013)$, para as notas de corte de 49 e 47 pontos. Verificou-se moderada associação entre a EEB e o TUGT nos três grupos $(\mathrm{p}<0,001) .{ }^{?}$

Um estudo retrospectivo comparou ${ }^{14}$ a $\mathrm{S}$ e E da EEB e TUGT em 17 idosos com histórico de uma queda em seis meses, 22 com quedas múltiplas (mais de uma em seis meses) e 39 sem quedas em três anos. Entre idosos com uma queda e sem quedas, o teste TUGT apresentou $\mathrm{S}=58,8 \%$ e $\mathrm{E}=88,2 \%$; entre caidores recorrentes e não caidores, $S=77,3 \%$ e $E=88,2 \%$; e entre uma queda e múltiplas quedas, $\mathrm{S}=88,2 \%$ e $\mathrm{E}=81,8 \%$. A escala EEB apresentou maior capacidade de discriminar as populações $(S=88,2 \%$ entre uma queda e nenhuma queda; $95,5 \%$ entre caidores recorrentes e não caidores; $94,1 \%$ entre uma queda e múltiplas quedas e $\mathrm{E}=76,5 \%$ entre uma queda e nenhuma queda; $95,5 \%$ entre caidores recorrentes e não caidores; $90,9 \%$ entre uma queda e múltiplas quedas).$^{14}$

Um estudo ${ }^{16}$ examinou a EEB e o TUGT em predizer quedas relacionadas a deslizes em 119 idosos saudáveis. O TUGT foi capaz de diferenciar os caidores de não caidores, com nota de corte 8,5s. O tempo acima de 7,6 segundos teve $\mathrm{S}=56 \%$ e $\mathrm{E}=60 \%$ em predizer quedas. $\mathrm{O}$ TUGT mostrou melhor capacidade preditiva que a EEB, possivelmente pelo efeito teto da EEB. ${ }^{16}$

Aoyama et al..$^{15}$ estudaram o TUGT e EEB na predição de quedas em 58 mulheres idosas (média 80,5 anos): 25 idosas caíram ao mínimo uma vez no período, enquanto 33 não sofreram quedas. Não houve diferença significativa entre caidores e não caidores nos dois testes. Boulgarides et al. verificaram se a EEB e o TUGT poderiam prever quedas em 99 idosos ativos e independentes. Não houve diferença significativa na EEB entre os indivíduos que não relataram queda $(57,6 \%)$ e os que relataram ao menos uma queda $(42,4 \%)$. O TUGT também não predisse quedas e o tempo dos não caidores encontrou-se entre 8 e 10s, e dos múltiplos caidores (maior ou igual a duas quedas), entre 9 e $13 .{ }^{17}$

A revisão mostrou que tanto a EEB quanto o TUGT podem ser bons preditores de quedas. No entanto, a presente revisão apresenta algumas limitações, a saber: carência de estudos prospectivos, variações nos estudos em relação à quantidade e características dos voluntários, dificuldade de definir caidor e caidores múltiplos e carência de avaliações cegas, dentre outros fatores. Alguns estudos uniformizaram a amostra quanto à ocorrência de eventos únicos (possíveis quedas acidentais) e quedas recorrentes (duas ou mais), devido aos mecanismos e fatores de risco para suas ocorrências serem diferentes. Observa-se, portanto, uma heterogeneidade nos estudos incluídos, tanto da amostra estudada quanto das formas de avaliação, o que justifica os diferentes resultados quanto aos pontos de corte, predição de quedas, sensibilidade, especificidade e eficácia dos testes. 


\section{CONCLUSÃO}

Com base nesta revisão, conclui-se que os testes Berg Balance Scale (EEB) e Timed Up and Go Test (TUGT) são eficazes para predição de quedas, desde que adaptados de maneira correta para cada perfil do idoso (por exemplo, TUGT-

\section{REFERÊNCIAS}

1. Lebrão ML, Duarte YAO, organizadoras. SABE: saúde, bem-estar e envelhecimento: o Projeto Sabe no município de São Paulo: uma abordagem inicial. Brasília: Organização Pan-Americana da Saúde 2003. $255 \mathrm{p}$.

2. Pereira SRM, Buksman S, Perracini M, Py L. Barreto KML, Leite VMM. Quedas em Idosos. [s.1.]: Sociedade Brasileira de Geriatria e Gerontologia; [2001?]. 9 p. (Projeto Diretrizes).

3. MMWR: Recommendations and reports. CDC Recommendations Regarding Selected Conditions Affecting Women's Health. Atlanta: Centers for Disease Control and Prevention, USA. Vol. 49, No. RR-2, Marc 2000 - .

4. Gillespie LD, Gillespie WJ, Robertson MC, Cordeiro $\mathrm{SE}$, Rowe BH. Interventions for preventing falls in elderly people. 2003. In: The Cochrane Database Systematic Reviews [Internet]. Hoboken, NJ: John Willey \& Sons, Ltd. c2003 -. [cerca de 44 p.]. Disponível em: http://cochrane.bvsalud.org/ cochrane/show.php?db $=$ reviews $\& \mathrm{mfn}=\& \mathrm{id}=\mathrm{CD} 000$ $340 \&$ lang $=\&$ dblang $=\&$ lib $=$ COC $\&$ print $=$ yes Registro No. CD000340.

5. Réseau Francophone de Prévention des Traumatismes et de Promotion de la Sécurité. Good Practice Guide: prevention of falls in the elderly living at home. SaintDenis: INPES; 2008.

6. Hofheinz M, Schusterschitz C. Dual task interference in estimating the risk of falls and measuring change: a comparative, psychometric study of four measurements. Clinical Rehabilitation 2010;24(9):831-42.

7. Thomas JI, Lane JV. A pilot study to explore the predictive validity of 4 measures of falls risk in frail elderly patients. Arch Phys Med Rehabil 2005;86(8):1636-40.

8. Deutsches Netzwerk fur Qualitatsentwicklung in der Pflege, hrsg. Expertenstandard Sturzprophylaxe in der Pflege: entwicklung, konsentierung, implementierung. Osnabruck: DNQP; 2006. 166 p.

9. Gonçalves DF, Ricci NA, Coimbra AM. Equilíbrio funcional de idosos da comunidade: comparação
DT e aumento da nota de corte da EEB para idosos ativos).

É necessário que novos estudos sejam realizados com metodologia e amostra homogênea, favorecendo a comparação de resultados e avaliação da eficácia desses testes.

em relação ao histórico de quedas. Rev Bras Fisioter 2009;13(4):316-23.

10. Alfieri FM, Carreres MAA, Figuera RG, Battistella LR. Comparación del tiempo de ejecución del test timed up and go (TUG) en ancianos con y sin antecedentes de caídas. Rev Esp Geriatr Gerontol 2010;45(3):172-5.

11. Chiu AYY, Au-Yeung SSY, Lo SK. A comparison of four functional tests in discrimination fallers from non-fallers in older people. Disabil Rehabil 2003;25(1):45-50.

12. Aoyama M, Suzuki Y, Onishi J, Kazuya M. Physical and functional factors in activities of daily living that predict falls in community-dwelling older women. Geriatr Gerontol Int 2011;11(3):348-57.

13. Bhatt T, Espy D, Yang F, Pai YC. Dynamic gait stability, clinical correlates, and prognosis of falls among community-dwelling older adults. Arch Phys Med Rehabil 2011;92(5)799-805.

14. Boulgarides LK, McGinty SM, Willett JA, Barnes $\mathrm{CW}$. Use of clinical and impairment-based tests to predict falls by community-dwelling older adults. Phys Ther 2003;83(4):328-39.

15. Herman T, Giladi N, Hausdorff JM. Properties of the 'timed up and go' test: more than meets the eye. Gerontol 2011;57(3):203-10.

16. Greene BR, O’Donovan A, Romero-Ortuno R, Cogan L, Scanaill CN., Kenny RA. Quantitative falls risk assessment using the timed up and go test. IEEE Trans Biomed Eng 2010;57(12):2918-26.

17. Wang CY, Hsieh CL, Olson SL, Wang CH, Sheu CF, Liang CC. Psychometric Properties of the Berg Balance Scale in a Community-dwelling Elderly Resident Population in Taiwan. J Formos Med Assoc 2006;105(12):992-1000.

18. Pimentel RM, Scheicher ME. Comparison of fall risk between sedentary and active aged by means of the Berg Balance Scale. Fisioter Pesqui 2009;16(1):6-10.

19. Lajoie Y, Gallagher SP. Predicting falls within the elderly community: comparison of postural sway, reaction time, the Berg Balance Scale and the 
Activities-specific Balance Confidence (ABC) scale for comparing fallers and non-fallers. Arch Gerontol Geriatr 2004;38(1):11-26.

20. Lajoie Y, Girard A, Guay M. Comparison of the reaction time, the Berg Scale and the $\mathrm{ABC}$ in nonfallers and fallers. Arch Gerontol Geriatr 2002; 35(3):215-25.

21. Bogle Thorbahn LD, Newton RA. Use of the Berg Balance Test to predict falls in elderly persons. Phys Ther 1996;76(6):576-83.

22. Lin MR, Hwang HF, Hu MH, Wu HD, Wang YW, Huang FC. Psychometric comparisons of the timed up and go, one-leg stand, functional reach, and tinetti balance measures in community-dwelling older people. J Am Geriatr Soc 2004;52(8):1343-8.

23. Shimada H, Tiedemann A, Lord SR, Suzukawa M, Makizako H, Kobayashi K, et al. Physical factors underlying the association between lower walking performance and falls in older people: a structural equation model. Arch Gerontol Geriatr 2011;53(2):131-4.

24. Rossat A, Fantino B, Nitenberg C, Annweiler C, Poujol L, Herrmann FR, et al. Risk factors for falling in community-dwelling older adults: which of them are associated with the recurrence of falls? J Nutr Health Aging 2010;14(9):787-91.

25. Shimada H, Suzukawa M, Tiedemann U, Kobayashi K, Yoshida H, Suzuki T. Which neuromuscular or cognitive test is the optimal screening tool to predict falls in frail community-dwelling older people? Gerontol 2009;55(5):532-8.

26. Miyamoto ST, Lombardi Jr. I, Berg KO, Ramos LR, Natour J. Brazilian Version of the Berg Balance Scale. Braz J Med Biol Res 2004;37(9):1411-21.

27. Berg KO, Wood-Dauphinee SL, Williams JI, Maki B. Measuring balance in the elderly: validation of an instrument. Can J Public Health 1992;83 Suppl 2:S7-11.

28. Soares KV, Figueiredo KMOB, Caldas VVA, Guerra RO. Avaliação quanto à utilização e confiabilidade de instrumentos de medida do equilíbrio corporal em idosos. Rev Publica 2005;1:78-85.

29. Steffen T, Hacker TA, Mollinger L. Age- and genderrelated test performance in community-dwelling elderly people: Six-Minute Walk Test, Berg Balance Scale, Timed Up \& Go Test, and Gait Speeds. Phys Ther 2002;82(2):128-37.

30. Rodini C, Ferreira LTD, Pirré GE, Hino M, Alfieri FM, Roberto M, et. al. Estudo comparativo entre a
Escala de Equilíbrio de Berg, o Teste Timed Up \& Go e o Índice de Marcha Dinâmico quando aplicadas em idosos hígidos. Acta fisiatra 2008;15(4):267-68.

31. Viccaro LJ, Perera S, Studenski SA. Is timed up and go better than gait speed in predicting health, function, and falls in older adults? J Am Soc Geriatr 2011;59(5):887-92.

32. Pai YC, Wang E, Espy DD, Bhatt T. Adaptability to perturbation as a predictor of future falls: a preliminary prospective study. J Geriatr Phys Ther 2010;33(2):50-5.

33. Aslan UB, Cavlak U, Yagci N, Akdag B. Balance performance, aging and falling: a comparative study based on a Turkish sample. Arch Gerontol Geriatr 2008;46(3):283-92.

34. Thrane G, Joakimsen RM, Thornquist E. The association between timed up and go test and history of falls: the Tromso study. BMC Geriatr 2007; 7(1):1-7.

35. Huang HC, Gau ML, Lin WC, George K. Assessing risk of falling in older adults. Public Health Nurs 2003;20(5):399-411.

36. Giné-Garriga M, Guerra M, Marí-Dell'Olmo M, Martin C, Unnithan VB. Sensitivity of a modified version of the 'timed get up and go' test to predict fall risk in the elderly: a pilot study. Arch Gerontol Geriatr 2009; 49(1):e60-6.

37. Donoghue D, Stokes EK; Physiotherapy Researche and Older People (PROP) Group. How much change is true change? The minimum detectable change of the Berg Balance Scale in elderly people. J Rehabil Med 2009;41(5):343-6.

38. Southard V, Dave M, Davis MG, Blanco J, Hofferber A. The multiple tasks test as a predictor of falls in older adults. Gait Posture 2005;22(4): 351-5.

39. Muir SW, Berg K, Chesworth B, Speechley M. Use of the Berg Balance Scale for predicting multiple falls in community-dwelling elderly people: a prospective study. Phys Ther 2008;88(4):449-59.

40. Ersoy Y, MacWalter RS, Durmus B, Altay ZE, Baysal O. Predictive effects of different clinical balance measures and the fear of falling on falls in postmenopausal women aged 50 years and over. Gerontology 2009;55(6):660-5.

41. Santos GM, Souza AC, Virtuoso JF, Tavares GM, Mazo GZ. Predictive values at risk of falling in physically active and no active elderly with Berg Balance Scale. Rev Bras Fisioter 2011;15(2):95-101. 